\section{Automatización en el hogar: Un proceso de diseño para viviendas de interés social}

FECHA DE RECEPCIÓN: 10 de enero FECHA DE APROBACIÓN: 2 de febrero Pp. 108-121

Boris Andrés Quintana G.* Vietnam Rafaela Pereira Poveda** Cindy Nayid Vega S. ** $^{*}$

Automation at home : a design

process for sacial low

cost houses

La domotique : une nouvelle

conception pour /e logement

d'intérêt social

Automatizaçãa no lar: um

processa de desenho para

moradias de interesse sacial

*Doctor (c) en Proyectos de Desarrollo Sostenible. Universidad Internacional Iberoamericana, México. Master Cambio Global Recursos Naturales y Sostenibilidad. Universidad de Córdoba, España.

Diseñador Industrial. Universidad Nacional de Colombia. Bogotá, Colombia.

Actualmente es docente investigador de la Fundación Universidad Autónoma de Colombia y es Director del Centro de Estudios Interdisciplinarios para el Desarrollo - CEIDE -

**Master in Business Administration (MBA) en Gestión Sostenible. Universidad Leuphana - Luneburgo, Alemania. Bióloga. Universidad Nacional de Colombia. Investigadora en el Centro de Estudios Interdisciplinarios para el Desarrollo - CEIDE ***Estudiante de Maestría en Ingeniería Industrial con énfasis en Investigación y gestión de las organizaciones. Universidad Distrital Francisco fosé de Caldas. Ingeniera Industrial. Universidad Autónoma de Colombia. Investigadora de la Fundación Universidad Autónoma de Colombia y del Centro de Estudios Interdisciplinarios para el Desarrollo - CEIDE -. 


\section{RESUMEN}

El presente artículo, presenta una investigación basada en el desarrollo de un sistema empleado para la ayuda a viviendas de bajos recursos por medio de sistemas domóticos. Tradicionalmente, han sido diseñados y elaborados para grupos de elevado poder adquisitivo, sin considerarse la responsabilidad ambiental y social de su producción e instalación. Es por ello que se realizó un estudio en el que se lograron identificar todos estos factores que aumentan el costo, con el fin de establecer la posibilidad de involucrar proyectos domóticos en viviendas de la población menos favorecida, a fin de implementar tecnología de autoproducción energética para el diseño y fabricación de objetos que contribuyan a la concientización del desperdicio energético del ser humano, y por otra más fundamental, al desarrollo económico en este grupo de base social. El trabajo no solo fue investigativo sino también de campo, el cual se verá identificado más adelante, con el fin de presentar este sistema de forma asequible para la comunidad mencionada.
(1) Palabras clave

Sistemas domóticos Viviendas

Autoproducción energética Sostenibilidad

\begin{abstract}
This article aims at showing a research study based on the development of a system used to support social low cost houses by using domotic systems. Through time, these systems have been designed for wealthy people without taking into account environmental and social responsibility in their manufacturing and installation. For this reason, a serious research was done to identify the factors which raise cost, trying to execute domotic projects in low cost houses to finally implement energetic self-generation technologies for the design and manufacturing of objects which contribute to make people conscious about energetic waste and the economic development of this low social class groups. Consequently, this research study not only involved scientific investigation but also field work, which will be described in this paper aiming at showing the above mentioned system for this social group.
\end{abstract}

\section{Keywords}

Domotic systems

Energetic waste Environmental and social Responsibility 


\section{RESUMÉE}

Cet article présente les résultats d'une recherche basée sur la mise en place d'un système utilisé pour venir en aide aux foyers à faible revenu par le biais des technologies domotiques. Traditionnellement conçus pour des populations à fort pouvoir d'achat, les systèmes domotiques ne prennent que très peu en compte la responsabilité sociale et environnementale de la production et de l'installation de ces technologies. Notre étude cherche à identifier tous les facteurs qui participent à l'augmentation du coût final des installations domotiques pour que ce type de projets puisse se réaliser dans des foyers défavorisés. Ces nouvelles technologies permettent en outre la réalisation d'économies d'énergie lors de la conception et de la fabrication d'objets contribuant à la conscientisation du respect à l'environnement chez cette classe sociale peu favorisée.

\section{RESUMO}

O artigo, apresenta uma pesquisa baseada no desenvolvimento de um sistema empregado para a ajuda a moradias de baixos recursos por meio de sistemas domóticos. Tradicionalmente, foram desenhadas e elaboradas para grupos de elevado poder aquisitivo, sem considerar a responsabilidade ambiental e social de sua produção e instalação. É por isso que se realizou um estudo no qual se conquistou identificar todos esses fatores que aumentam o custo, com o fim de estabelecer a possibilidade de envolver projetos domóticos em moradias da população menos favorecida, com a finalidade de implementar tecnologia de auto produção energética para o desenho e fabricação de objetos que contribuíam a conscientização do desperdício energético do ser humano e por outro lado ainda mais fundamental, ao desenvolvimento econômico neste grupo de base social. Por esse motivo que o trabalho não foi somente de pesquisa científica mas também foi realizada uma pesquisa de campo, a qual se verá identificada mais adiante, com o fim de apresentar esse sistema de forma acessível para a comunidade mencionada.
Mots clefs

Systèmes domotiques

Responsabilité

environnementale

Dépense énergétique.

\section{Q Palavras-chave}

Sistemas domóticos

Desperdício energético

Responsabilidade ambiental. 


\section{INTRODUCCIÓN}

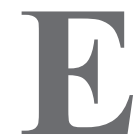

I desarrollo domótico, en su contexto tradicional y su lenta entrada en el contexto colombiano por la barrera económica, ha generado una pregunta inicial acerca de la posibilidad de la aplicación de energías renovables para su funcionamiento. Sin embargo, la respuesta a este interrogante obedece a los intereses, tanto económicos como de satisfacción, de necesidades de los grupos sociales de alto poder adquisitivo.

De esta reflexión surge un cuestionamiento sobre la responsabilidad social que deberían mantener las

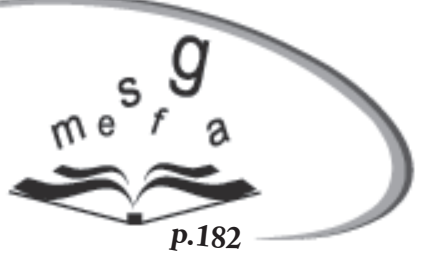
empresas, concerniente a la fabricación e instalación de dichos sistemas, puesto que el concepto domótica no se entiende en el propio contexto como una tecnología que puede potenciar el bienestar humano y del medio ambiente global, sino como un conjunto de prestaciones suntuosas de automatización de dispositivos en el hogar que amplía los niveles de confort de quienes la adquieren.

Este proyecto se apoya en una postura similar, en la cual y desde el desarrollo tecnológico se prospecta su apropiación popular, en especial desde las poblaciones menos favorecidas. Circunscribiendo dicho problema a la ciudad de Bogotá, para delimitarle, y teniendo en cuenta una variable importante como lo es el capítulo verde en términos de sostenibilidad integral del proyecto, se definió una pregunta base de investigación: ¿Es posible realizar una

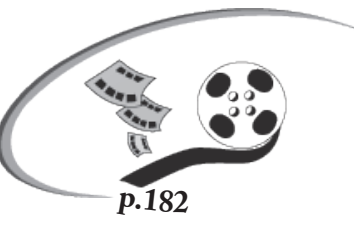
aplicación domótica sostenible para viviendas de interés social en Bogotá?

Para encontrar la respuesta a este cuestionamiento, fue necesario aterrizar la investigación a una población en Bogotá, para lo cual se escogieron las urbanizaciones OASIS IV (Suba) y Bochica 6 y 5, Etapa II (Engativá), en el Noroccidente de Bogotá, ambas Vivienda de Interés Social (VIS).

Aparte de las limitantes de localización, se tuvieron en cuenta otras en el transcurso del proyecto, como fueron: la delimitación tecnológica: sistemas domóticos con sistemas de operación por sensor; la delimitación socio-económica y ambiental: sostenibilidad global en un marco normativo en Bogotá; y la delimitación de mercado: usuarios directos de las (VIS).

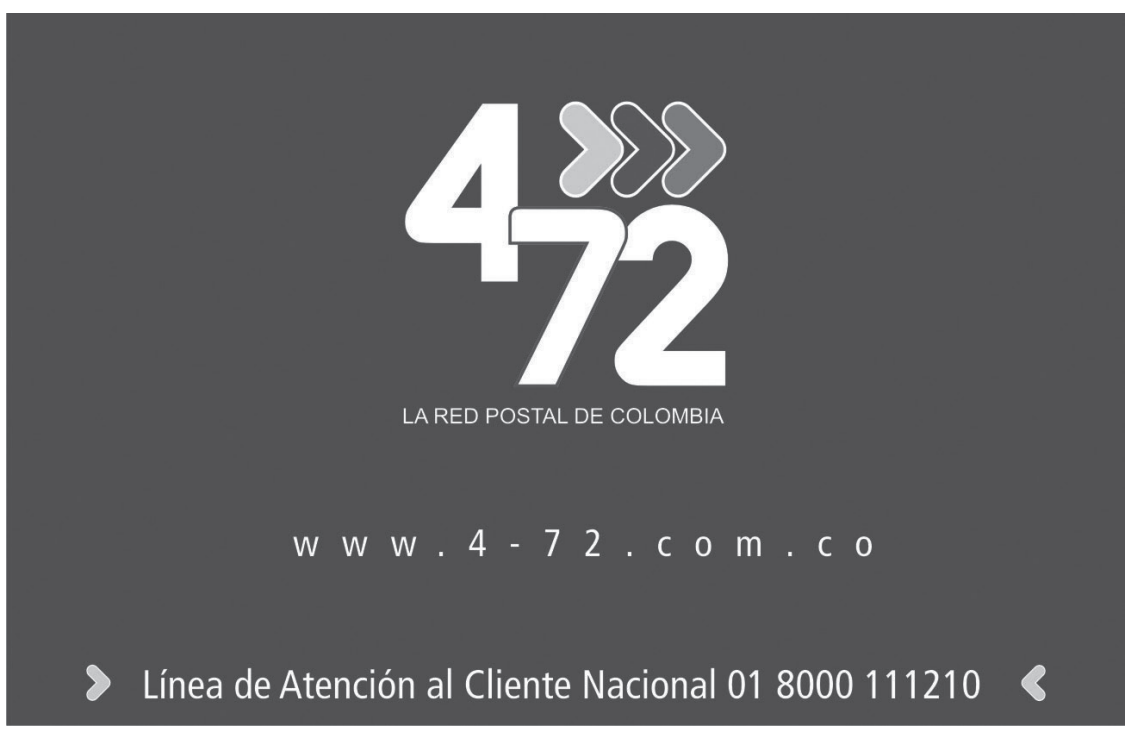




\section{METOdOLOGÍA}

E I procedimiento metodológico que permite el desarrollo del proyecto formulado por el Centro de Estudios Interdisciplinarios para el Desarrollo (CEIDE) (grupo Investigador), emplea como herramientas metodológicas:

- Levantamiento de información secundaria (información bibliográfica).

- Levantamiento de información primaria (aplicación de encuestas a productores, constructores, organismos públicos y usuarios), para el cual se diseñaron las encuestas, y una evaluación minuciosa de las plataformas Web más indicadas para la aplicación de las mismas, según las necesidades específicas del proyecto.

- Análisis de resultados de las encuestas.

- Análisis de Ciclo de Vida del prototipo a diseñar e implementar, durante el periodo de prueba, incluyendo todas las variables del análisis

\section{CONCEPUALIZACIÓN DEL PROYECTO}

$\mathbf{P}$ ara consolidar epistemológicamente el proyecto, fue necesario recabar información desde varios núcleos temáticos, siendo estos la Vivienda de Interés Social (VIS), la domótica y la producción alternativa de energía eléctrica.

En cuanto al primer concepto, la vivienda en términos simples, es definida como uno de los derechos sociales y económicos, según las Naciones Unidas, a través de diversos tratados y declaraciones de derechos humanos que han sido ratificados por Colombia, como aquella que se desarrolla para garantizar el derecho a una solución de vivienda, entendida esta como el conjunto de operaciones que permite a un hogar disponer de habitación en condiciones sanitarias satisfactorias de espacio, servicios públicos y calidad de estructura, o iniciar el proceso para obtenerlas en el futuro .

Estas soluciones de vivienda, están disponibles en Colombia para hogares con ingresos bajos, que varían dependiendo de la ciudad de asentamiento y van desde aquellos iguales o inferiores a dos Salarios Mínimos
Legales Vigentes (SMLV), hasta aquellos iguales o inferiores a cuatro SMLV. Adicionalmente a los ingresos, los hogares que deseen acceder a las VIS, deben cumplir con una serie de requisitos tendientes a garantizar que la asignación de los subsidios sea para hogares que realmente los necesiten.

Para entender el concepto de vivienda digna en el cual se quiere enmarcar el proyecto, se debe dar cumplimiento a las Normas Colombianas de Diseño y Construcción, de acuerdo con la Ley 400 de 1997.

El segundo concepto, el de domótica, término acuñado en español y posterior a su referente anglo-sajón House Automation, proviene del latín domus: casa; y automática: funcionamiento autónomo. Hace referencia a la incorporación, en la vivienda, de un conjunto de tecnologías informáticas y de comunica-ciones que permiten gestionar y automatizar, desde un mismo sistema, las diferentes instalaciones de uso cotidiano, proporcionando una mejor calidad de vida de los usuarios y una mejor conservación y cuidado del edificio. 
La domótica se caracteriza por ofrecer los siguientes cinco aspectos:

- Gestión energética: ahorro en el consumo y reducción de emisiones, tales como dióxido de carbono $\left(\mathrm{CO}^{2}\right)$, dióxido de azufre $\left(\mathrm{SO}^{2}\right)$, precursor de la lluvia ácida (Universidad de Sevilla, 2011, p. 13).

- Confort: todas las actuaciones que incrementan la comodidad en una vivienda. Estas pueden ser pasivas, activas o mixtas.

- Seguridad: red encargada de proteger, tanto los bienes patrimoniales, como la seguridad personal.

- Comunicaciones: interconexión de la red domótica con diferentes dispositivos como Internet, telefonía fija y móvil, etc., lo que permite telemantenimiento, informes de consumo y costos, intercomunicaciones, teleasistencias, entre otros servicios.

- Accesibilidad: aplicaciones o instalaciones de control remoto del entorno, que benefician la autonomía personal de individuos con limitaciones funcionales o discapacidad.

\subsection{Ejemplos de domótica global}

Actualmente, a nivel mundial se están construyendo edificios más automatizados, permitiendo ver dicha automatización como un servicio casi básico de toda edificación, a la par que se viene presentando la tendencia a la protección del medio ambiente.

En Madrid, la empresa española Home Sistems, ha automatizado hasta el año 2012, 450 viviendas, cuya solución domótica cubre la gestión de seguridad, control, detección de fuga de gas o agua e iluminación, utilizando el sistema X10.

En Oriente, la torre biónica en Hong Kong, es un proyecto de los arquitectos españoles Eloy Celaya, Javier Gómez Pioz y María Rosa Cervera. Será una torre-ciudad vertical, con $1.228 \mathrm{~m}$ de altura e inspirada en el tronco de los árboles, la cual tendrá a su vez, una estructura de anillos y raíces. Cien mil personas podrán habitar en esta edificación que contará con oficinas, guarderías, colegios, etc. y un puente que la enlazará con la ciudad.

La casa inteligente en San Fernando, es una casa que fue transformada en un hogar inteligente, al estar habitada por una persona discapacitada. Es una instalación de control electrónico integral de energía limpia, mediante placas fotovoltaicas que funcionan como control de encendido y apagado; control de electrodomésticos desde un punto central; gestiona el control de persianas, toldos y puertas individualmente, con un temporizador o control de alarmas técnicas. Estos controles se pueden realizar por voz y se indican mediante un sonido o luz de confirmación.

También, la catedral de Múnich en Alemania, ha sido modernizada por los miembros de LONMARK. Se pueden monitorear todos los componentes como el control de luz, el sistema de alarma, la vigilancia por cámara y el control de las lámparas desde un punto central. Esta integración de componentes fue lograda con monitores de contacto de 19" instalados en la sacristía. 


\section{CONCEPUALIZACIÓN DEL PROYECTO}

$\mathbf{L}$

os resultados de las encuestas practicadas a los usuarios actuales de VIS, permiten concluir en primera instancia, que el desconocimiento en cuanto a temas de sostenibilidad y domótica, es de casi el $50 \%$ de la población objetivo del estudio.

Una vez hecha una breve explicación sobre los dispositivos domóticos y la relación de su uso con la sostenibilidad a nivel del hogar, se indagó sobre el interés de las familias en utilizar este tipo de elementos en su hogar. El resultado de esta indagación, permite ver que el interés en el uso doméstico de dispositivos domóticos asciende apenas al 63\%, lo cual se relaciona de manera directa con el desconocimiento de los temas, lo que a su vez, genera desconfianza en la utilización de dispositivos nuevos cuyo funcionamiento se desconoce.

Dentro de la población, dada la poca o nula información que se tiene sobre temas como tecnología de punta, domótica o sostenibilidad, se ve claramente la tendencia a asumir que las nuevas tecnologías son costosas o de difícil manejo, y que la sostenibilidad es un asunto de responsabilidad gubernamental muy alejado del día a día de una familia promedio colombiana. Así mismo, se asume que los costos de adquisición y mantenimiento de tecnología de punta son elevados, lo que la hace inaccesible para hogares con ingresos inferiores a cuatro salarios mínimos legales.

Teniendo en cuenta la poca relación que la población objetivo ve con los temas de sostenibilidad y de usos de tecnología de punta, se indagó sobre la relación

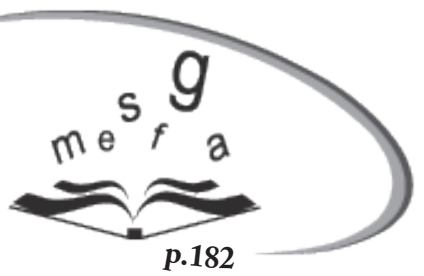
que los usuarios encuentran entre el uso de dispositivos de esta última tecnología, con el consumo de energía reflejado en la factura de servicios públicos, encontrando que para el $92 \%$ de los usuarios, es clara la relación directa entre el uso de cualquier dispositivo con los consumos de recursos, representados en las facturaciones de los servicios. Se trata de un factor de preocupación permanente en hogares que financieramente dependen de los salarios de uno o de varios de los miembros de la familia. Es importante resaltar que esta incidencia no está estimada como beneficiosa o contraproducente, sólo en si existe o no.

Así como se aprecia que para los usuarios es claro que el uso de tecnologías está directamente relacionado con su factura de energía eléctrica, también es claro que la tecnología está directamente relacionada con aspectos de la calidad de vida en el hogar. Al indagar sobre la percepción de los usuarios en cuanto al tipo de relación entre estos aspectos, se devela nuevamente la falta de información acerca de las tecnologías como herramientas de mejoramiento de la calidad de vida y la sostenibilidad, como concepto de desarrollo personal y social.

Aunque la mayoría de los usuarios considera que el uso de domótica en sus hogares implicaría mejorar su calidad de vida, no es claro para todos ellos en qué se vería representada esta mejora en términos concretos. La mayoría lo interpreta como un asunto de comodidad en disminución de las labores del hogar, no como un factor de reducción de costos recurrentes, o de aprovechamiento eficiente de los recursos.

Después de indagar sobre la relación que puede tener el uso de dispositivos domóticos con la calidad de vida, se investigó sobre la relación entre el uso de este tipo de tecnología con el medio ambiente; el resultado concluyó lo siguiente:

- Se mantiene el imaginario colectivo de que la tecnología, independientemente de sus características, afecta negativamente el medio ambiente.

- No se ve relación directa entre el medio ambiente y la calidad de vida de las personas; se concibe al Ser humano como un ente completamente ajeno a la naturaleza y sus interacciones. 
Dentro de los impactos ambientales negativos que el grueso de la población conoce, existe la tendencia a considerar la generación de emisiones como la consecuencia más importante del uso de tecnologías en el día a día. En los resultados de esta consulta, también es claro que el conocimiento de la población objetivo sobre impactos ambientales, es bastante reducido.

Otro de los sectores entrevistados, teniendo en cuenta los objetivos del presente trabajo, fue el de la administración pública. En este sector, se consultó sobre los conocimientos en cuanto al tema de domótica y la factibilidad de su utilización en las VIS en Colombia.

En este orden de ideas, se consultó una población compuesta por funcionarios del nivel nacional (40\%) y del nivel distrital en Bogotá (60\%). Dentro de esta muestra, el $40 \%$ de los entrevistados hacen parte del sector vivienda y territorio y el $60 \%$ del sector ambiente y desarrollo sostenible.

Al investigar sobre el nivel de conocimiento en domótica por parte de los entrevistados, se encontró que sólo el 40\% manifiesta tener conocimientos sobre el tema; a su vez, de este porcentaje, sólo el $40 \%$ considera que es factible la implementación de este tipo de tecnología en las VIS, en la jurisdicción en la que trabajan.

Teniendo en cuenta que el porcentaje de apreciación negativa sobre la factibilidad de implementación de domótica en las VIS es del $60 \%$ de la población entrevistada, se establecieron las consideraciones al respecto, encontrando que no existe uniformidad de criterio ni tendencia en el tema; de acuerdo con lo anterior, es posible inferir que no existe un lineamiento gubernamental clara.

Considerando que una de las causas por la que los servidores públicos no ven factibilidad en la implementación de domótica en VIS es el costo de su instalación, se indagó sobre la relación costo-beneficio del uso de los mismos, obteniendo como resultado la claridad de que esta relación es beneficiosa para el usuario (67\%), si bien no es calificada como excelente (33\%), obtiene unánimemente evaluaciones positivas. De esta observación se concluye que, a pesar de la positiva relación costo-beneficio que se tiene para el usuario final de los dispositivos domóticos, a nivel gubernamental es clara la preocupación en cuanto al costo de adquisición para entrega a usuarios, sin una tasa de retorno al erario público.

Considerando la claridad que se tiene en cuanto a los beneficios económicos que la domótica reportaría a los usuarios, se indagó sobre la relación que puede tener con otros aspectos de la calidad de vida de estas personas, obteniendo que para todos, es claro que el uso de este tipo de tecnologías aporta al mejoramiento de esta. Con base en las respuestas encontradas, se preguntó a la población objetivo si consideraban la incorporación de este tipo de dispositivos en las VIS como elementos de gobernabilidad, presentando como resultado que la mayoría (67\%) no ven una relación entre la gobernabilidad y la inversión social que representa la entrega de VIS con este tipo de tecnologías instaladas.

El porcentaje más reducido, que considera que estas iniciativas llevadas a la práctica se constituyen en un elemento de gobernabilidad, estima que serían incluibles dentro de las especificaciones técnicas de los proyectos, jugándole así también al desarrollo y la transparencia que proclama el gobierno nacional.

Finalmente, se evalúa la percepción que tienen los servidores públicos sobre el impacto ambiental que tienen los dispositivos domóticos de uso doméstico, obteniendo que el $67 \%$ considera que estos generan impactos ambientales negativos en cuanto a generación de residuos. Así como se percibe que los dispositivos domóticos generarían residuos no gestionables en el país, también es claro que implicaría un aumento significativo en la eficiencia del uso del recurso energético. 


\section{APLICACIÓN DEL SISTEMA DOMÓTICO}

E

n conclusión, con los resultados obtenidos de las encuestas realizadas a la población, se definió el diseño y construcción de un sistema domótico (ducha), que beneficiara a la población; puesto que los costos que implicó esta construcción fueron bajos y el tiempo invertido para el primer prototipo fue de seis meses, en este tiempo se evaluaron los materiales, el diseño y la puesta en marcha del producto, a una vivienda de la población descrita anteriormente. A continuación se presentan las primeras imágenes de las partes del prototipo elaborado por el grupo de investigación (Figura 1).

Figura 1. Carcasa anterior
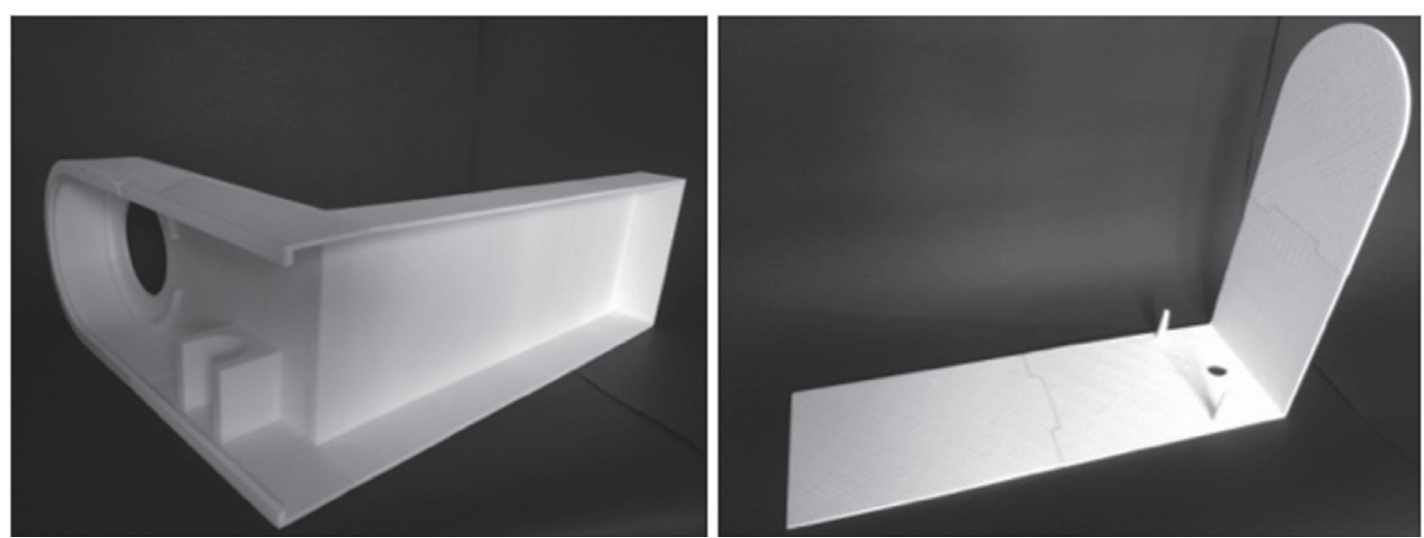

Fuente. Elaboración propia de los autores, 2014.

Tal como se aprecia en la carcasa interior cual soporta los claves, la entrada del agua es la parte que protege todo el sistema.

A continuación se aprecia la tapa en donde están albergados los cables y el sistema hidráulico; la imagen de la derecha muestra la pieza que soporta la tapa del sistema domótico (Figura 2).

Figura 2. Tapa Unidad A (unidad hidráulica)
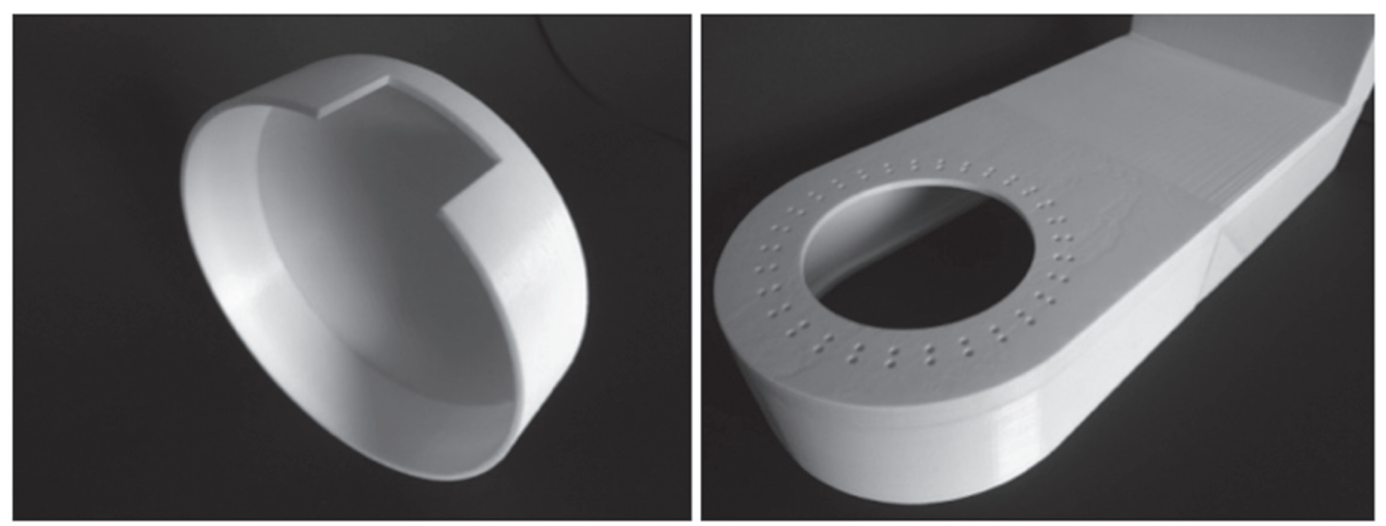

Fuente. Elaboración de los autores, 2014.

Posteriormente, se muestra el tubo de entrada y la tapa de las aspas que cubre y protege el sistema de agua que ingresa en el producto (Figura 3). 
Figura 3. Tubo de entrada y tapa aspas
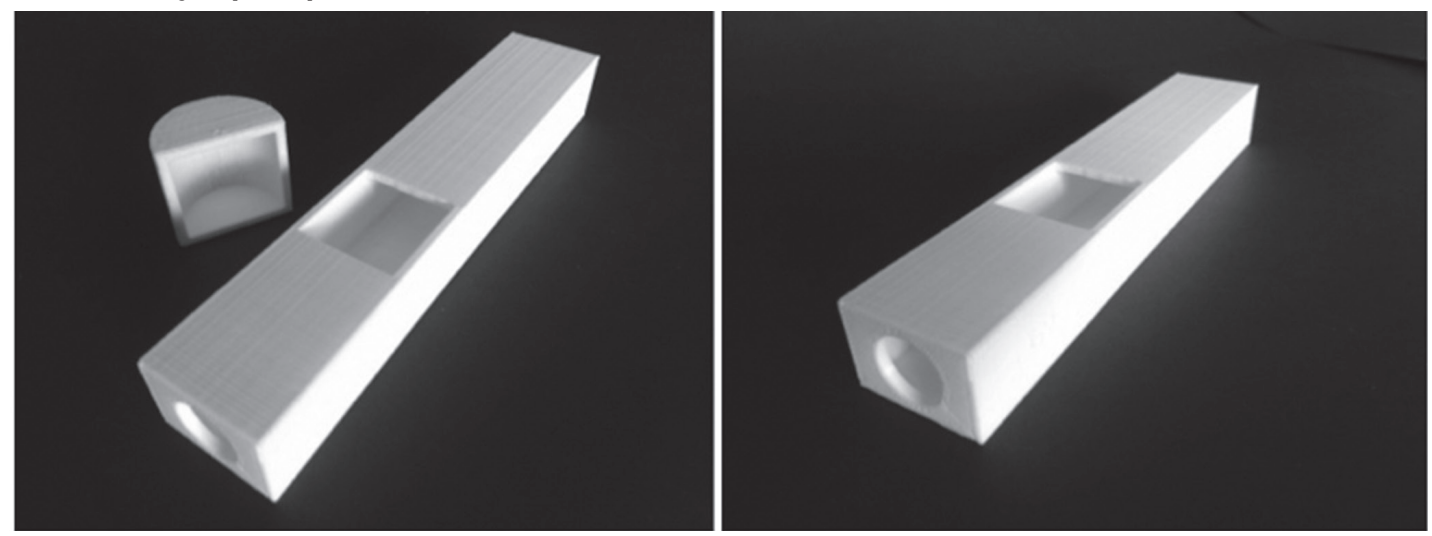

Fuente. Elaboración propia de los autores, 2014.

Ahora, se verá la puesta de los leds de colores que tienen un trabajo definido dentro del sistema domótico (Figura 4).

\section{Figura 4. Tapa Unidad B}
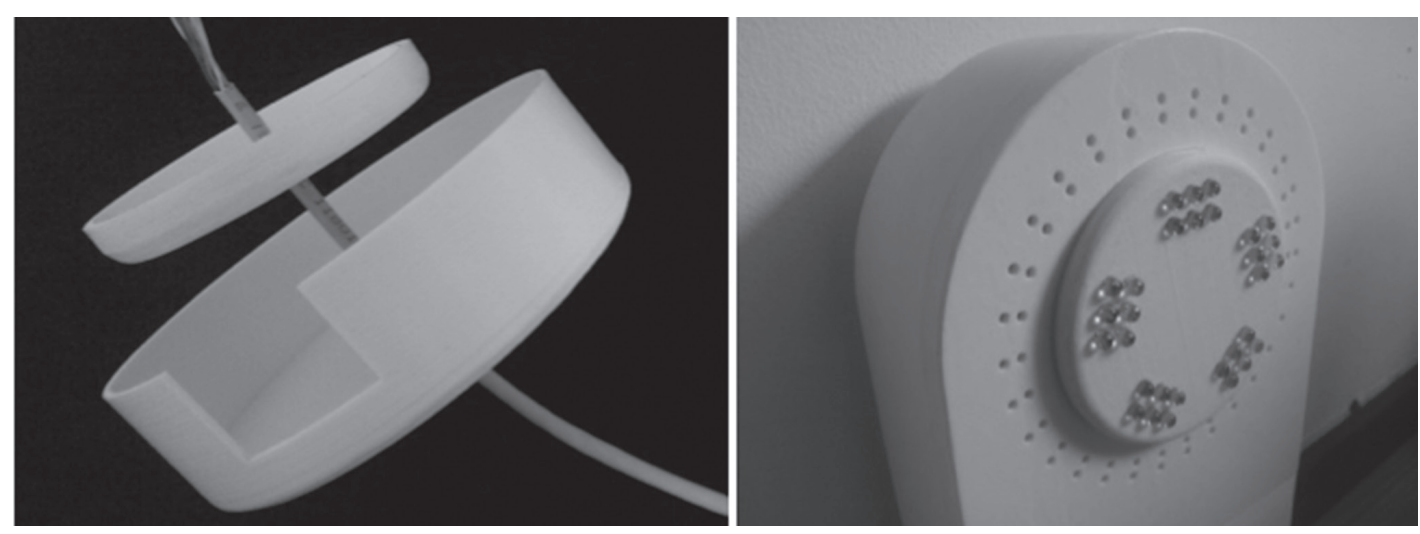

Fuente. Elaboración propia de los autores, 2014.

Por último, se indica el sistema implementado en una casa ubicada en la localidad de Suba en la ciudad de Bogotá (Figura 5).

Figura 5. Proyecto final en funcionamiento

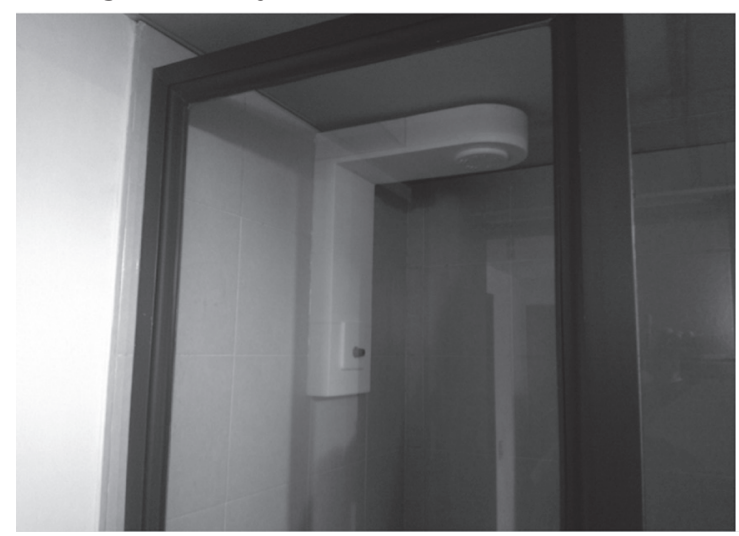

Fuente. Elaboración propia de los autores, 2014. 
Como se puede evidenciar en el proceso anterior, el sistema domótico construido para los habitantes de Viviendas de Interés Social (VIS), es un producto de uso diario para el aseo integral de cada habitante. Esta construcción, además de ser ambiental y económicamente sostenible, aporta a las personas un sistema que provee beneficios lumínicos relacionados con energía física y mental; como se puede constatar, es un sistema bastante dinámico que puede ser mejorado y utilizado para otros productos que se diseñen por medio de estas técnicas.

\section{CONCLUSIONES}

E I uso de energía residual humana como fuente de energía para dispositivos domésticos, puede ser una solución para proveer a los habitantes de una vivienda confort, seguridad, abastecimiento de iluminación y abastecimiento de energía eléctrica para necesidades puntuales, como la carga de dispositivos electrónicos pequeños.

Adecuadamente aprovechada y cosechada, la energía residual humana puede convertirse en un sustituto parcial del abastecimiento de red de energía eléctrica, en las VIS de la ciudad de Bogotá.

Con base en los resultados obtenidos en las encuestas, se concluye que el conocimiento general en la población usuaria de VIS, con respecto a estas tecnologías, es bajo; las personas tienen nociones muy vagas y erradas en cuanto a las nuevas tecnologías, por lo cual la mayoría asocia directamente el uso de tecnología con la generación de emisiones y la contaminación ambiental.
Los resultados también revelan que los servidores públicos tienen un buen nivel de conocimiento de las tecnologías, a pesar de no identificar el término "domótica", es decir, saben de las tecnologías, entienden sus aplicaciones y aplicabilidades, tienen nociones muy certeras de sus usos, pero no conocen el término técnico que las define.

Una vez evacuado el punto del conocimiento de las tecnologías o su aproximación a ellas, y analizando los demás puntos de la encuesta, se concluye que tanto para la población objetivo, como para el Gobierno, la posibilidad de implementar este tipo de tecnologías en poblaciones de bajos recursos económicos, es interesante, tanto en términos de economía familiar, como de confort.

La implementación de este sistema, es bastante beneficioso para las familias de bajos recursos, ya que es de bajo costo y provechoso para la salud de las personas de mayor edad y niños. El sistema domótico seguirá sus ajustes para que continúe el posible proceso de fabricación y distribución. 


\section{REFERENCIAS BIBLIOGRÁFICAS}

Aristizabal, A J. (2004). Dimensionamiento e instalación de un sistema fotovoltaico interconectado a la red eléctrica y desarrollo de equipo para analizar calidad de potencia, empleando LABVIEW. (Trabajo de grado, Universidad Nacional de Colombia, Manizales).

Asociación Española de Domótica - CEDOM \& Instituto para la Diversificación y Ahorro de la Energía (IDAE). (2008). Cómo ahorrar energía instalando domótica en su vivienda, gane en confort y seguridad. Recuperado de: http:// www.cedom.es/fitxers/documents/publicacions_home/Guia\%20de\%20Ahorro\%20Energetico\%20CEDOM. pdf /

Azcárate, B.M. (2008). Energías e impacto ambiental. Madrid, España: Ed. Sirius.

Bounzas, M J. (2005). Panorámica de los sistemas domóticos e inmóticos. (Trabajo de grado, Escuela Superior de Ingenieros).

Díaz Reyez, C. (2011). Los materiales en la construcción de vivienda de interés social, Aincol. (Guías de Asistencia Técnica para Vivienda de Interés Social). Segunda cartilla. Colombia: Ministerio de Ambiente, Vivienda y Desarrollo Territorial.

DINASTER. Noticias (2011). Ingeniería domótica recibe el Premio a la mejor instalación domótica. Recuperado de: http://www.dinaster.com/Noticias-Lujo/ingenieria-domotoca-recibe-el--premio-a-la-mejor-instalacion -domotica-0694.html /

Domínguez, H M. \& Sáez, F. (2006). Domótica, un enfoque sociotécnico. Madrid: Fundación Rogelio Segovia para el Desarrollo de las Telecomunicaciones.

DOMÓTICA. Noticias (2011). Las ventajas del uso de la domótica. Recuperado de: http://www.domoticausuarios.es/ las-ventajas-del-uso-de-la-domotica/2602/

Duque, A \& Ulloa, Y. (2012). Diseño de un sistema de juguetes generadores de energía eléctrica proveniente de la energía humana disipada. (Tesis de grado, Fundación Universidad Autónoma de Colombia).

EDUCARED. (2011). Situación actual de la domótica. Recuperado de: http://www.educared.org/global/anavegar5/ podium/images/D/2476/intr_04.htm

Fondo Europeo de Desarrollo Regional MetalTIC-Hogar digital. (2011). El Proyecto. Recuperado de: www.metaltic. org/

FUNDACIÓN PRIVADA INSTITUT ILDEFONS CERDÀ. (2000). Ahorro, confort, seguridad y comunicaciones, la vivienda Domótica. Barcelona, España. Ministerio de Industria y Energía, Dirección General de Industrias y Tecnologías de la Información. 
García, E. \& López, F. J.(2004). El estado del arte de la tecnología al servicio de la construcción. España: (s.e).

González, J.A. (2009). Centrales de energías renovables. Madrid: Ed. Prentice Hall.

Harper, R. (2003). Inside the Smart Home: Ideas, Possibilities and Methods. London: Springer.

Herrera, L. F. (2004). Gestión tecnológica para domótica y telecontrol. (Trabajo de grado de maestría en Telecomunicaciones, Universidad Nacional de Colombia, Bogotá, D.C.).

Huidobro, J. M. y Millán, R. J. (2004). Domótica Edificios Inteligentes. España: Creaciones Copyright.

Jansen, A.J. (2011). Human power Empirically Explored. Holanda: Delft University of Technology.

Ingeniería Domótica. Noticias ingeniería. (2010). Domótica recibe el Premio a la mejor instalación Domótica 2010 por una vivienda en Somosagua. Recuperado de: http://www.ingenieriadomotica.com/noticia3.swf/

Méndez, F. J. (2010). La domótica: nuevas formas de entender la vivienda. En: Directivos Construcción, 239, 48.

Ortega, C. A., Roque, D. y Asbeda, L. (2008). Zigbee: El nuevo estándar global para la domótica e inmótica. Recuperado de: http://www.monografias.com/trabajos-pdf/zigbee/zigbee.pdf/

Quintero, L. F. (2005). Viviendas inteligentes (domótica). En: Revista Ingeniería e Investigación. Universidad Nacional de Colombia, 25(002). pp. 47-53.

Requero, A. (1998). Estado actual y perspectivas de la domótica. Recuperado de: http://informesdelaconstruccion. revistas.csic.es/index.php/informesdelaconstruccion/article /view/827/912 /

Salazar, A., Bruchi, et al. (2003). Automatización. Recuperado de: http://sifunpro.tripod.com/automatizacion.htm/

Sartor, A. (s.f). La tecnología y el ambiente. Recuperado de: http://www.frbb.utn.edu.ar/carreras/materias/ing_soc/ tecnologia_y_ambiente.pdf.

Volkow, N. (2003). La brecha digital, un concepto social con cuatro dimensiones. En: Inegi. Boletín de Política Informática, 6.

\section{Documentos Jurisprudenciales}

Alcaldía Mayor de Bogotá. Decreto 061/2003, por el cual se adopta el Plan de Gestión Ambiental del Distrito Capital.

Alcaldía Mayor de Bogotá. Decreto 456/2008, por el cual se reforma el Plan de Gestión Ambiental del Distrito Capital y se dictan otras disposiciones.

Alcaldía Mayor de Bogotá. Plan de desarrollo 2012 - 2016, Bogotá Humana.

Congreso de la República de Colombia. Ley 3 de 1991, por la cual se crea el Sistema Nacional de Vivienda de Interés Social, se establece el subsidio familiar de vivienda, se reforma el Instituto de Crédito Territorial, ICT, y se dictan otras disposiciones. 
Congreso de la República de Colombia. Constitución Política de Colombia. 1991.

Departamento Nacional de Planeación, República de Colombia. Plan Nacional de Desarrollo 2010 - 2014, Prosperidad para todos.

Ley 697 de 2001 Recuperdado de: http://www.lawea.org/documentos/Colombia_Ley_697.pdf

Ministerio de Ambiente, Vivienda y Desarrollo Territorial, Ministerio de Minas y Energía. Decreto 2501 de 2007. Por medio del cual se dictan disposiciones para promover prácticas con fines de uso racional y eficiente de energía eléctrica.

Presidencia de la República. Decreto 1168 de 1996, por el cual se reglamentan parcialmente la Ley 3a de 1991 y la Ley 60 de 1993, en materia de subsidios municipales para vivienda de interés social.

Presidencia de la República. Ley 1450 de 2011, por medio de la cual se expide el Plan Nacional de Desarrollo 2010 - 2014. 